Article

\title{
Coordinate Scaling in Time-Independent Excited-State Density Functional Theory for Coulomb Systems
}

\author{
Ágnes Nagy \\ Department of Theoretical Physics, University of Debrecen, H-4010 Debrecen, Hungary; anagy@phys.unideb.hu \\ Received: 26 September 2019; Accepted: 11 October 2019; Published: 13 October 2019 \\ check for \\ updates
}

\begin{abstract}
A time-independent density functional theory for excited states of Coulomb systems has recently been proposed in a series of papers. It has been revealed that the Coulomb density determines not only its Hamiltonian, but the degree of excitation as well. A universal functional valid for any excited state has been constructed. The excited-state Kohn-Sham equations bear resemblance to those of the ground-state theory. In this paper, it is studied how the excited-state functionals behave under coordinate scaling. A few relations for the scaled exchange, correlation, exchange-correlation, and kinetic functionals are presented. These relations are expected to be advantageous for designing approximate functionals.
\end{abstract}

Keywords: density functional theory; excited states; Coulomb systems; coordinate scaling

\section{Introduction}

Density functional theory (DFT) was originally developed for the ground state [1,2]. The first exact theories were the subspace theory of Theophilou [3] and the ensemble theory of Gross, Oliveira, and Kohn [4]. Later, it turned out that it is possible to construct a theory for a single excited state, e.g., utilizing the density of the given state and the external potential [5,6]. It has been shown that in case of Coulomb systems the density of the given state is enough to characterize the state $[7,8]$. A time-independent density functional theory for excited states of Coulomb systems has recently been proposed in a series of papers [9-11]. It has been revealed that the Coulomb density determines not only its Hamiltonian but the degree of excitation as well. A universal functional valid for any excited state has been constructed. The excited-state Kohn-Sham equations bear resemblance to those of the ground-state theory. Even the generalization for degenerate excited states has been done.

The exact form of the exchange-correlation functional is not known even for the ground state. One needs approximation to perform calculations. It turned out that exact relations are useful in constructing approximate functionals for the ground state. It is supposed that this statement is true for excited states as well. Via coordinate scaling, one can derive exact constraints that the excited-state functionals should satisfy. These relations are expected to be advantageous for designing approximate forms of excited-state functionals. Coordinate scaling has proved to be very important in DFT. It was first proposed by Ghosh and Parr [12] and Levy and Perdew [13]. Later, a lot of valuable relations were derived via coordinate scaling (see, e.g., [14-26]). For example, Liu and Parr [24] proposed a linear combination of homogeneous functionals of different specific degrees in coordinate scaling for the correlation energy. In this paper, some exact scaling expressions are derived within the new theory. These expressions are the same as in the ground-state theory.

The paper is organized as follows: In Section 2 the excited-state DFT for Coulomb systems [9-11] is summarized. Section 3 presents a few exact relations for the scaled exchange, correlation, exchange-correlation, and kinetic functionals. Section 4 is dedicated to discussion. 


\section{Excited-State DFT for Coulomb Systems}

Coulomb systems are very common, as atoms, molecules, and solids are all Coulomb systems. The Hamiltonian can be written as

$$
\hat{H}=\hat{T}+\hat{V}_{e e}+\sum_{i=1}^{N} v^{\operatorname{Coul}}\left(\mathbf{r}_{i}\right),
$$

where $\hat{T}$ and $\hat{V}_{e e}$ are the kinetic energy and the electron-electron energy operators, respectively.

$$
v^{\mathrm{Coul}}(\mathbf{r})=-\sum_{\beta=1}^{M} \frac{Z_{\beta}}{\left|\mathbf{r}-\mathbf{R}_{\beta}\right|},
$$

where $R_{\beta}$ denotes the position of the nucleus $\beta$ with charge $Z_{\beta}$ and $M$ is the number of nuclei. The density of the Coulomb system is sufficient in principle to determine all of its properties. The cusps of the density tell us where the nuclei are and what the atomic numbers are and the integral of the density yields the number of electrons. It is true for excites states as well and excited-state theory can be formulated utilizing this statement $[7,8]$. It has been proved that the electron density of a Coulomb system cannot be a stationary state density for any other Coulomb external potential and two different excited states cannot have the same electron density [9].

Construct now the fuctional

$$
F^{\operatorname{Coul}}[n]=E_{k}[n]-\int n(\mathbf{r}) v^{\operatorname{Coul}}[n ; \mathbf{r}] d \mathbf{r},
$$

where $n$ is the density of a stationary state of any Coulomb system. However, there is no known way to decide whether a given density is Coulombic or not (without constructing the external potential and solving the Schrödinger equation). Therefore, it is better to define a functional $F$ for all electron densities.

Define first a bifunctional

$$
F\left[n, n^{\text {Coul }}\right]=\min _{\substack{\left.\Psi \rightarrow n \\\left\{\langle\Psi| \Psi_{l}^{\text {Coul }}[n \text { Coul }]\right\rangle=0\right\}_{l=1}^{k-1}}}\left\langle\Psi\left|\hat{T}+\hat{V}_{e e}\right| \Psi\right\rangle
$$

The minimization of the kinetic plus electron-electron energies is done with the constraint that each wave function gives the excited-state density $n$ and is orthogonal to the first $k-1$ eigenfunctions of the Coulomb system fixed by $n^{\mathrm{Coul}}$. Assume that there exists a unique Coulomb density that is closest to the (non-Coulomb) density $n$. If there are several Coulomb densities that are the same distance from $n$, the one leading to the smallest $F$ in Equation (4), is selected.

$$
F_{\epsilon}^{\text {Coul }}[n]=\min _{n^{\text {Coul }}} F\left[n, n^{\text {Coul }}\right] ; \quad\left\|n^{\text {Coul }}-n\right\| \leq \epsilon .
$$

$\epsilon$ is supposed to be large enough to ensure the existence of at least one stationary state Coulomb density in the distance smaller than $\epsilon$. Finally, we take the $F$ with the smallest $\epsilon$ :

$$
F^{\text {Coul }}[n]=F_{\epsilon_{\text {min }}}^{\text {Coul }}[n] .
$$

Turn now to the non-interacting kinetic energy

$$
T_{S}\left[n^{\text {Coul }}\right]=\min _{\substack{\Phi \rightarrow n^{\text {Coul }} \\\left\{\left\langle\Phi \mid \Phi_{l}\left[n^{\text {Coul }}\right]\right\rangle=0\right\}_{l=1}^{k-1} \\\left\|n_{1}^{\text {Coul }}-n_{1}^{0}\right\| \leq \delta}}\langle\Phi|\hat{T}| \Phi\rangle .
$$


According to this definition the ground-state density $n_{1}^{0}$ of the non-interacting system should be as close as possible to the ground-state density of the original Coulomb system $n_{1}^{\text {Coul }}$. Then a bifunctional is defined as

$$
T_{s}^{\text {Coul }}\left[n, n^{\text {Coul }}\right]=\min _{\substack{\Phi \rightarrow n \\\left\{\left\langle\Phi \mid \Phi_{l}\left[n^{\text {Coul }}\right]\right\rangle=0\right\}_{l=1}^{k-1} \\\left\|n_{1}^{\text {Coul }}-n_{1}^{0}\right\| \leq \delta}}\langle\Phi|\hat{T}| \Phi\rangle
$$

After similar steps as above we are led to

$$
T_{s, \epsilon}^{\text {Coul }}[n]=\min _{n^{\text {Coul }}} T_{S}^{\text {Coul }}\left[n, n^{\text {Coul }}\right]
$$

where

$$
\left\|n^{\mathrm{Coul}}-n\right\| \leq \epsilon .
$$

The final expression of the definition of the non-interacting kinetic energy is

$$
T_{s}^{\text {Coul }}[n]=T_{s, \epsilon_{\text {min }}}^{\text {Coul }}[n] .
$$

Kohn-Sham equations can also be derived

$$
\left[-\frac{1}{2} \nabla^{2}+w^{\operatorname{Coul}}([n], \mathbf{r})\right] \phi_{i}=\varepsilon_{i} \phi_{i}
$$

where the Kohn-Sham potential

$$
w^{\operatorname{Coul}}([n], \mathbf{r})=v^{\operatorname{Coul}}([n], \mathbf{r})+v_{J}^{\operatorname{Coul}}([n], \mathbf{r})+v_{x c}^{\text {Coul }}([n], \mathbf{r})
$$

is the sum of the external, the classical Coulomb and the exchange-correlation potentials. The density has the form

$$
n=\sum_{i=1}^{I} g_{i}\left|\phi_{i}\right|^{2}
$$

where the occupation numbers $g_{i}$ are 0,1 or 2 for a non-degenerate system. $I$ denotes the orbital having the highest orbital energy with non-zero occupation number.

\section{Coordinate Scaling}

The (uniform) coordinate scaling means that the coordinates $\mathbf{r}_{i}$ are changed into $\alpha \mathbf{r}_{i}$, where $\alpha$ is any real constant. The wave function and the density scale as $\Phi_{n}^{\alpha}\left(\mathbf{r}_{1}, \ldots, \mathbf{r}_{N}\right)=\alpha^{3 N / 2} \Phi\left(\alpha \mathbf{r}_{1}, \ldots, \alpha \mathbf{r}_{N}\right)$ and $n_{\alpha}(\mathbf{r})=\alpha^{3} n(\alpha \mathbf{r})$, respectively.

One can derive a lot of scaling relations. Here, only a few are considered. These are expected to be important. Certainly, there are other significant scaling expressions that are not presented here.

The classical Coulomb energy

$$
J^{\operatorname{Coul}}[n]=\frac{1}{2} \int \frac{n\left(\mathbf{r}_{1}\right) n\left(\mathbf{r}_{2}\right)}{\left|\mathbf{r}_{1}-\mathbf{r}_{2}\right|} d \mathbf{r}_{1} d \mathbf{r}_{2}
$$

scales as usual

$$
J^{\operatorname{Coul}}\left[n_{\lambda}\right]=\lambda J^{\operatorname{Coul}}[n]
$$


with $\alpha=\lambda^{-1}$. The exchange energy can be expressed with the average of $\hat{V}_{e e}$

$$
E_{x}^{\operatorname{Coul}}[n]=\left\langle\Phi\left|\hat{V}_{e e}\right| \Phi\right\rangle-J^{\operatorname{Coul}}[n] .
$$

Note that in Equation (17) $\Phi$ is the non-interacting wave function defined by the steps described above (Equations (7)-(11)). One can readily see that

$$
E_{x}^{\text {Coul }}\left[n_{\lambda}\right]=\lambda E_{x}^{\text {Coul }}[n] .
$$

Similarly, the non-interacting kinetic energy $T_{s}^{\text {Coul }}[n]$ scales as

$$
T_{s}^{\text {Coul }}\left[n_{\lambda}\right]=\lambda^{2} T_{s}^{\text {Coul }}[n] .
$$

Turning to the scaling of the exchange-correlation energy, it is worth studying the operator $\hat{T}+$ $\alpha \hat{V}_{e e}$, because the scaled wave function $\Psi_{n}^{\alpha}\left(\mathbf{r}_{1}, \ldots, \mathbf{r}_{N}\right)$ yields $n$ and minimizes $\left\langle\hat{T}+\alpha \hat{V}_{e e}\right\rangle$, as described in Equations (4)-(6). $E_{x c}^{\text {Coul }, \alpha}[n]$ is defined as

$$
E_{x c}^{\text {Coul }, \alpha}[n]=\left\langle\Psi^{\alpha}\left|\hat{T}+\alpha \hat{V}_{e e}\right| \Psi^{\alpha}\right\rangle-\alpha J^{\operatorname{Coul}}[n]-T_{s}^{\text {Coul }}[n],
$$

where $\alpha=1$ gives the exchange-correlation energy $E_{x c}^{C o u l}[n]$, functional derivative of which appears in the Kohn-Sham potential (13). Then we immediately arrive at

$$
E_{x c}^{\text {Coul }, \alpha}[n]=\alpha^{2} E_{x c}^{\text {Coul }}\left[n_{\lambda}\right], \quad \alpha=\lambda^{-1},
$$

The correlation energy correcponding to the coupling constant $\alpha$ is defined in the same way as in the ground-state DFT (see, e.g., Equation (8) in [18])

$$
E_{c}^{\text {Coul }, \alpha}[n]=\left\langle\Psi^{\alpha}\left|\hat{T}+\alpha \hat{V}_{e e}\right| \Psi^{\alpha}\right\rangle-\left\langle\Psi^{0}\left|\hat{T}+\alpha \hat{V}_{e e}\right| \Psi^{0}\right\rangle,
$$

where $\Psi^{0}=\Phi$ is the non-interacting wave function. Taking into account the scaling of the exchange-correlation (Equation (21)) and exchange (Equation (18)) energies, we are led to the relation

$$
E_{c}^{\text {Coul }, \alpha}\left[n_{\lambda}\right]=\alpha^{2} E_{c}^{\text {Coul }}\left[n_{\lambda}\right] .
$$

From Equation (20) we immediatelly obtain

$$
\lim _{\alpha \rightarrow 0} \lambda^{-1} E_{x c}^{\text {Coul }}\left[n_{\lambda}\right]=\min _{n^{\text {Coul }}} \min _{\substack{\Psi \rightarrow n \\\left\{\Psi\left|\Psi_{l}^{\text {Coul }}\left[n^{\text {Coul }]}\right]\right\rangle=0\right\}_{l=1}^{k-1}}}\left\langle\Psi\left|\hat{V}_{e e}\right| \Psi\right\rangle-J^{\text {Coul }}[n] .
$$

Finally, consider the Hamiltonian with the coupling constant $\alpha$

$$
\hat{H}^{\alpha}=\hat{T}+\alpha \hat{V}_{e e}+\sum_{i=1}^{N} w^{\operatorname{Coul}, \alpha}\left(\mathbf{r}_{i}\right) .
$$

$w^{\text {Coul }, \alpha}$ is defined by keeping the density $n$ fixed. Note that $w^{\operatorname{Coul}, \alpha=1}=v^{\mathrm{Coul}}$ is the external potential of the original interacting Coulomb system (see Equation (2)) and $w^{\mathrm{Coul}, \alpha=0}=w^{\mathrm{Coul}}$ is the Kohn-Sham potential. Following the derivation in Reference [18], we arrive at the scaling expression:

$$
w^{\operatorname{Coul}, \alpha}([n], \mathbf{r})=w^{\operatorname{Coul}, 0}([n], \mathbf{r})-\alpha\left[v_{J}^{\operatorname{Coul}}([n], \mathbf{r})+v_{x}^{\operatorname{Coul}}([n], \mathbf{r})+\alpha \frac{\delta E_{\mathcal{C}}^{\operatorname{Coul}}\left(\left[n_{\lambda}\right]\right.}{\delta n(\mathbf{r})}\right] .
$$

The most interesting term in Equation (26) is last one. It mirrors the highly complicated nature of the correlation energy funcional. 


\section{Discussion}

In this paper Coulomb systems are considered, that is, the external potential is given by Equation (2). It is emphasized that only the true, original interacting external potential has the Coulomb form. The non-interacting potential, that is, the Kohn-Sham potential, does not have the structure of Equation (2). The superscript Coul in $w^{\text {Coul }}$ in Equation (13) indicates that $w^{\text {Coul }}$ is the Kohn-Sham potential constructed to describe a Coulomb system. It has to be also underlined that the external potential with coupling constant $\alpha$ does not have the shape of Equation (2) unless $\alpha=1$.

Unfortunately, the exact exchange-correlation functional is unknown. It is undiscovered even for the ground state. It is also unexplored in the excited-state theory of Coulomb systems presented here. However, it is needed for calculations. In the original ground-state DFT, several approximations are available for computation. Exact relations that the exact exchange-correlation functional satisfies proved to be useful in the construction of certain approximate functionals (see, e.g., [27].) The same benefit is expected in this excited-state theory. Here, some coordinate scaling relations are presented that are supposed to be helpful in modeling excited-state functionals.

We defined $F^{\mathrm{Coul}}[n]$ that depend only on the density. It is valid for any excited state and for the ground state, too. We do not need different functionals for different excited states. One $F^{\mathrm{Coul}}[n]$ is sufficient for any state of a Coulomb system. However, it might happen that $F^{\text {Coul }}[n]$ is discontinous. Therefore, functionals $F_{k}^{\text {Coul }}[n]$ that depend on the level of excitation, $k$, have also been defined. Discontinuities are less likely in $F_{k}^{\mathrm{Coul}}[n]$ (see details in [9]). Here, it is supposed that $F^{\mathrm{Coul}}[n]$ and all other functionals defined here are well-behaved.

The coordinate scaling relations presented here are the same that were found previously for the ground state. It is not surprising because this theory is valid for both ground and excited states. We hope that an approximation suitable both for ground and excited states will be found in the future.

In conclusion, some exact relations are derived by means of coordinate scaling in the DFT theory of Coulomb systems. These relations are the same as in the ground-state DFT. Approximation valid both for ground and excited states is expected to be constructed later.

Funding: This research was supported by the National Research, Development and Innovation Fund of Hungary, financed under 123988 funding scheme.

Conflicts of Interest: The author declares no conflict of interest.

\section{References}

1. Hohenberg, P.; Kohn, W. Inhomogeneous Electron Gas. Phys. Rev. J. Arch. 1964, 136, B864. [CrossRef]

2. Kohn, W.; Sham, L.J. Self-Consistent Equations Including Exchange and Correlation Effects. Phys. Rev. J. Arch. 1965, 140, A1133. [CrossRef]

3. Theophilou, A.K. The energy density functional formalism for excited states. J. Phys. C Solid State Phys. 1979, 12, 5419. [CrossRef]

4. Gross E.K.U.; Oliveira, L.N.; Kohn, W. Density-functional theory for ensembles of fractionally occupied states. I. Basic formalism. Phys. Rev. A 1988, 37, 2809. [CrossRef] [PubMed]

5. Levy, M.; Nagy, Á. Variational Density-Functional Theory for an Individual Excited State. Phys. Rev. Lett. 1999, 83, 4361. [CrossRef]

6. Nagy, Á.; Levy, M. Variational density-functional theory for degenerate excited states. Phys. Rev. A 2001, 63, 052502. [CrossRef]

7. Nagy, Á. Optimized potential method for ensembles of excited states. Int. J. Quantum Chem. 1998, 69, $247-254$. [CrossRef]

8. Nagy, Á. Excited states in density functional theory. Int. J. Quantum Chem. 1998, 70, 681. [CrossRef]

9. Ayers, P.W.; Levy, M.; Nagy, Á. Time-independent density-functional theory for excited states of Coulomb systems. Phys. Rev. A 2012, 85, 042518. [CrossRef]

10. Ayers, P.W.; Levy, M.; Nagy, Á. Kohn-Sham theory for excited states of Coulomb systems. J. Chem. Phys. 2015, 143, 191101. [CrossRef] 
11. Ayers, P.W.; Levy, M.; Nagy. Á. Time-independent density functional theory for degenerate excited states of Coulomb systems. Theor. Chim. Account. 2018, 137, 152. [CrossRef]

12. Ghosh, S.K.; Parr, R.G. Density-determined orthonormal orbital approach to atomic energy functionals. J. Chem. Phys. 1985, 82, 3307. [CrossRef]

13. Levy, M.; Perdew, J.P. Hellmann-Feynman, virial, and scaling requisites for the exact universal density functionals. Shape of the correlation potential and diamagnetic susceptibility for atoms. Phys. Rev. A 1985, 32, 2010. [CrossRef] [PubMed]

14. Ou-Yang, H.; Levy, M. Theorem for exact local exchange potential. Phys. Rev. Lett. 1990, 65, 1036. [CrossRef]

15. Levy, M. Density-functional exchange correlation through coordinate scaling in adiabatic connection and correlation hole. Phys. Rev. A 1991, 43, 4637. [CrossRef]

16. Görling, A.; Levy, M.; Perdew, J.P. Expectation values in density-functional theory, and kinetic contribution to the exchange-correlation energy. Phys. Rev. B 1993, 47, 1167. [CrossRef]

17. Levy, M.; Perdew, J.P. Density functionals for exchange and correlation energies: Exact conditions and comparison of approximations. Int. J. Quantum Chem. 1994, 49, 539-548. [CrossRef]

18. Görling, A.; Levy, M. Correlation-energy functional and its high-density limit obtained from a coupling-constant perturbation expansion. Phys. Rev. B 1993, 47, 13105. [CrossRef]

19. Levy, M.; Perdew, J.P. Tight bound and convexity constraint on the exchange-correlation-energy functional in the low-density limit, and other formal tests of generalized-gradient approximations. Phys. Rev. B 1993, 48, 11638. [CrossRef]

20. Görling, A.; Levy, M. Exact Kohn-Sham scheme based on perturbation theory. Phys. Rev. A 1994, 50, 196. [CrossRef]

21. Levy, M.; Görling, A. Bounds for the exchange and correlation potentials. Phys. Rev. A 1995, 51, 2851. [CrossRef] [PubMed]

22. Levy, M.; Görling, A. New exact relations for improving the exchange and correlation potentials. Int. J. Quantum Chem. 1995, 56, 385-388. [CrossRef]

23. Levy, M.; Görling, A. Density-functional exchange identity from coordinate scaling. Phys. Rev. A 1996, 53, 3140. [CrossRef] [PubMed]

24. Liu, S.B.; Parr, R.G. Expansions of the correlation-energy density functional $E_{\mathcal{c}}[\varrho]$ and its kinetic-energy component $T_{\mathcal{C}}[\varrho]$ in terms of homogeneous functionals. Phys. Rev. A 1996, 53, 2211. [CrossRef] [PubMed]

25. Liu, S.B.; Nagy, Á.; Parr, R.G. Expansion of the density-functional energy components $E_{\mathcal{C}}$ and $T_{\mathcal{C}}$ in terms of moments of the electron density. Phys. Rev. A 1999, 59, 1131. [CrossRef]

26. Nagy, Á.; Liu, S.B.; Parr, R.G. Density-functional formulas for atomic electronic energy components in terms of moments of the electron density. Phys. Rev. A 1999, 59, 3349. [CrossRef]

27. Sun, J.W.; Ruzsinsky, A.; Perdew, J.P. Strongly Constrained and Appropriately Normed Semilocal Density Functional. Phys. Rev. Lett. 2015, 115, 036402. [CrossRef]

(C) 2019 by the author. Licensee MDPI, Basel, Switzerland. This article is an open access article distributed under the terms and conditions of the Creative Commons Attribution (CC BY) license (http:/ / creativecommons.org/licenses/by/4.0/). 